\title{
Experimental infection with H1N1 European swine influenza virus protects pigs from an infection with the 2009 pandemic H1N1 human influenza virus
}

\author{
Núria Busquets ${ }^{1 \dagger}$, Joaquim Segalés ${ }^{1,2 \dagger}$, Lorena CóRdobA ${ }^{1}$, Tufaria Mussé ${ }^{1}$, \\ Elisa CRISCI ${ }^{1}$, Gerard E. Martín-VAlls ${ }^{1}$, Meritxell SimON-GrifÉ ${ }^{1}$, \\ Marta Pérez-Simó ${ }^{1}$, Monica Pérez-Maíllo ${ }^{1}$, Jose I. NúÑEz ${ }^{1}$, Francesc X. AbaD ${ }^{1}$, \\ Lorenzo Fraile ${ }^{1,3}$, Sonia Pina ${ }^{1,3}$, Natalia Majó ${ }^{1,2}$, Albert Bensaid ${ }^{1}$, \\ Mariano Domingo ${ }^{1,2}$, María Montoya ${ }^{1,3 *}$ \\ ${ }^{1}$ Centre de Recerca en Sanitat Animal (CReSA), UAB-IRTA, Campus de la Universitat Autònoma de Barcelona, \\ 08193 Bellaterra, Barcelona, Spain \\ ${ }^{2}$ Departament de Sanitat i d'Anatomia Animals, Universitat Autònoma de Barcelona (UAB), 08193 Bellaterra, \\ Barcelona, Spain \\ ${ }^{3}$ Institut de Recerca i Tecnologia Agroalimentàries (IRTA), Barcelona, Spain
}

(Received 3 February 2010; accepted 26 July 2010)

\begin{abstract}
The recent pandemic caused by human influenza virus A(H1N1) 2009 contains ancestral gene segments from North American and Eurasian swine lineages as well as from avian and human influenza lineages. The emergence of this $\mathrm{A}(\mathrm{H} 1 \mathrm{~N} 1) 2009$ poses a potential global threat for human health and the fact that it can infect other species, like pigs, favours a possible encounter with other influenza viruses circulating in swine herds. In Europe, H1N1, H1N2 and H3N2 subtypes of swine influenza virus currently have a high prevalence in commercial farms. To better assess the risk posed by the A(H1N1) 2009 in the actual situation of swine farms, we sought to analyze whether a previous infection with a circulating European avian-like swine A/Swine/Spain/53207/2004 (H1N1) influenza virus (hereafter referred to as SwH1N1) generated or not cross-protective immunity against a subsequent infection with the new human pandemic A/Catalonia/63/2009 (H1N1) influenza virus (hereafter referred to as $\mathrm{pH} 1 \mathrm{N1}$ ) 21 days apart. Pigs infected only with $\mathrm{pH} 1 \mathrm{~N} 1$ had mild to moderate pathological findings, consisting on broncho-interstitial pneumonia. However, pigs inoculated with SwH1N1 virus and subsequently infected with pH1N1 had very mild lung lesions, apparently attributed to the remaining lesions caused by SwH1N1 infection. These later pigs also exhibited boosted levels of specific antibodies. Finally, animals firstly infected with SwH1N1 virus and latter infected with $\mathrm{pH} 1 \mathrm{~N} 1$ exhibited undetectable viral RNA load in nasal swabs and lungs after challenge with $\mathrm{pH} 1 \mathrm{~N} 1$, indicating a cross-protective effect between both strains.
\end{abstract}

influenza virus / swine H1N1 / human A(H1N1) 2009 / cross-protection

\section{INTRODUCTION}

Influenza viruses belong to the Orthomyxoviridae family and are characterized by a seg- mented viral genome consisting of eight single stranded RNA fragments of negative polarity encoding 10 proteins [27]. In April 2009, a new A(H1N1) influenza virus was

\footnotetext{
${ }^{\dagger}$ N. Busquets and J. Segalés equally contributed to the studies presented in this paper.

* Corresponding author: maria.montoya@cresa.uab.es
} 
identified containing a distinct combination of gene segments from both North American and Eurasian swine influenza lineages as well as from avian and human lineages [8, 21], which rapidly reached pandemic proportions. Most human infections with this new swine-origin H1N1 influenza virus variant, $\mathrm{A}(\mathrm{H} 1 \mathrm{~N} 1)$ 2009, seem to be mild; however, there was a substantial number of hospitalized young individuals without previous underlying health problems, attesting to the pathogenic potential of A(H1N1) 2009 in humans.

Influenza viruses circulating in swine are closely related to the human $\mathrm{H} 1 \mathrm{~N} 1$ and $\mathrm{H} 3 \mathrm{~N} 2$ strains and reports of sporadic cross-species transfer of swine and avian influenza viruses to humans have been documented repeatedly during recent decades [18]. The mortality of swine influenza virus (SIV) infected pigs is usually low, although morbidity may approach $100 \%$ [14]. Swine influenza is characterized by sudden onset, coughing, respiratory distress, weight loss, fever, nasal discharge and rapid recovery [14]. Epithelial cells in the swine respiratory tract have receptors for both avian and mammalian influenza viruses [12]; thus, pigs could potentially serve as "mixing vessels" for the generation of new reassortant strains.

Currently, few studies have assessed the effect of the new pandemic A(H1N1) 2009 in pigs. No signs of disease were observed in miniature pigs infected with $\mathrm{A}(\mathrm{H} 1 \mathrm{~N} 1)$ 2009, although it replicated efficiently in the respiratory tract of these animals. According to this study, the asymptomatic infection, despite efficient virus replication, might explain why this new reassortant has never been found in swine before it was first identified in humans [13]. Another study analyzed replication dynamics, clinical symptoms and virus transmission in pigs infected with the novel A(H1N1) 2009. The inoculated pigs started nasal virus shedding from day 1 post-inoculation (PI) onwards and developed generally mild symptoms including fever, sneezing, nasal discharge, and diarrhoea. In that study, contact pigs became infected, shed virus and developed clinical symptoms similar to the inoculated animals [16].
The emergence of this novel human influenza virus A(H1N1) 2009 poses a potential global threat for human health. Since the new virus can infect other species, like pigs, a possible encounter with other influenza viruses circulating in swine herds may favour the possibility of generating new reassortants with higher virulence. To date, there are several reports confirming the diagnosis of $\mathrm{A}(\mathrm{H} 1 \mathrm{~N} 1) 2009$ pandemic influenza virus in pig herds in all continents ${ }^{1}$. In all cases, the herds are believed to have been infected as a result of human-to-pig transmission. Therefore, the possibility that this novel human influenza virus A(H1N1) 2009 could affect a high percentage of swine herds has unknown consequences, not only for animal health but also for human health. A crucial question is whether previous immunity to circulating SIV protects pigs against pandemic A(H1N1) 2009 virus. Kyriakis et al. [17] have recently shown that pigs dually infected with some European SIV frequently exhibit crossreactive hemagglutination inhibitory $(\mathrm{HI})$ antibodies to pandemic A(H1N1) 2009 virus and related North American SIV, suggesting that pigs in Europe may have partial immunity to the pandemic $\mathrm{A}(\mathrm{H} 1 \mathrm{~N} 1) 2009$ virus. The question of whether these antibodies would prevent an $\mathrm{A}(\mathrm{H} 1 \mathrm{N1}) 2009$ infection remained unanswered. Thus, the objectives of the current study were (i) to experimentally investigate whether or not a previous infection with circulating H1N1 European avian-like swine influenza would confer protection to pigs which later encounter the novel pandemic human influenza virus $\mathrm{A}(\mathrm{H} 1 \mathrm{~N} 1) 2009$ and (ii) to further explore the pathological and immunological parameters of this new A(H1N1) 2009 virus infection in pigs. The present study shows that a single exposure to a H1N1 European avian-like swine influenza protects pigs against a consecutive challenge with the pandemic $\mathrm{A}(\mathrm{H} 1 \mathrm{~N} 1) 2009$ virus even in the absence of previous detectable cross-reactive $\mathrm{HI}$ antibodies.

\footnotetext{
${ }^{1}$ OIE, http://www.oie.int/eng/normes/mmanual/2008/ pdf/2.08.08_SWINE_INFLUENZA.pdf..
} 


\section{MATERIALS AND METHODS}

\subsection{Viruses}

Two Influenza A virus isolates were used in this study: the European avian-like swine A/Swine/ Spain/53207/2004 (H1N1) isolated in 2004 (GenBank accession number CY010587) (hereafter referred to as SwH1N1) and the new human $\mathrm{A} /$ Catalonia/63/2009 (H1N1) influenza virus isolated in 2009 (GenBank accession numbers GQ464405-GQ464411 and GQ168897) (hereafter referred to as pH1N1). SwH1N1 was generously donated by Laboratorios HIPRA (Spain) and it was propagated following standard procedures by infecting Madin-Darby canine kidney (MDCK) cells cultured in Dulbecco's modified Eagle's medium (DMEM) supplemented with $10 \%$ foetal bovine serum (FBS) (ref. 23 OIE). pH1N1 was isolated from a patient at the Hospital Clinic, Barcelona, Spain, and was propagated at $37.5^{\circ} \mathrm{C}$ in the allantoic cavities of 11 day-old embryonated chicken eggs originating from a commercial specific-pathogen-free (SPF) flock (GDdeventer) ${ }^{1}$. Both viruses were titrated in MDCK cells, with the aid of trypsin in the post-infection media, and virus titre was calculated by the Reed and Muench method [19].

\subsection{Animals}

Twenty-two snatch-farrowed, colostrum-deprived Large White $\times$ Landrace piglets were obtained from a herd with a standard health status. Sows were seropositive to porcine reproductive and respiratory syndrome virus (PRRSV) and SIV, and seronegative to Aujeszky's disease virus. Piglets were obtained at the moment of delivery, immediately dried and umbilical cords clamped, cut and disinfected with an iodine solution. Piglets were fed ad libitum during the first two days with the milk substitute Patavie Porc (Oriane-Celtilait, Lesneven, France). Afterwards, animals received Startrite 100 (SCA Ibérica S.A., Mequinenza, Spain) mixed with milk or as dry meal from 10-15 days of age. Antibiotics administered in the feed included 205000 UI of colistin ( $1 \mathrm{~g}$ of colimicine ${ }^{\circledR}$, Laboratorios SP Veterinaria, in $1.5 \mathrm{~L}$ of milk) during the first week of age and $1.7 \mathrm{mg}$ of enrofloxacin (Baytril oral solution $0.5 \%$, Bayer Animal Health, Leverkusen, Germany) per kg body weight/day during the first two weeks of age. All piglets were housed in an experimental isolation room at the biosafety level 3 facilities of the Centre de Recerca en Sanitat Animal (CReSA, Barcelona, Spain). Animal care and procedures were in accordance with the guidelines of the Good Laboratory Practices (GLP) and under the supervision of the Ethical and Animal Welfare Committee of the Universitat Autònoma de Barcelona.

\subsection{Experimental design}

At the age of 40 days, pigs were randomly distributed into four groups, namely Mock/Mock $(n=6), \quad$ SwH1N1/Mock $(n=4), \quad$ Mock/pH1N1 $(n=8)$ and SwH1N1/pH1N1 $(n=4)$ balanced by sex and weight. Group Mock/Mock pigs were intranasally inoculated with $3.5 \mathrm{~mL}$ of MEM (half of the amount in each nostril) on days 0 and 21 of the experiment. Group SwH1N1/Mock animals were intranasally inoculated with $3.5 \mathrm{~mL}$ of a suspension containing $10^{7.04}$ tissue culture infectious doses $50 \%\left(\mathrm{TCID}_{50}\right)$ per $\mathrm{mL}$ of SwH1N1 on day 0 and received the same volume of MEM on day 21. Pigs from group Mock/pH1N1 received the abovementioned amounts of MEM on day 0 and $10^{6.15} \mathrm{TCID}_{50}$ per $\mathrm{mL}$ of $\mathrm{pH} 1 \mathrm{~N} 1$ on day 21. Finally, pigs of group SwH1N1/pH1N1 were inoculated with both viruses, SwH1N1 on day 0 and pH1N1 on day 21, using the same route and dose mentioned for previous groups.

In order to study the events taking place at the early stages of infection with $\mathrm{pH} 1 \mathrm{~N} 1$, two Mock/Mock and two Mock/pH1N1 piglets were euthanized with an intravenous overdose of sodium pentobarbital on days 2 and 4 after pH1N1 inoculation (days 23 and $25 \mathrm{PI}$ ). All the remaining pigs in each group (2 animals of Mock/Mock group and 4 animals in each of the remaining groups) were euthanized on day $28 \mathrm{PI}$.

\subsection{Clinical records and sampling procedures}

Pigs were clinically monitored daily for the whole experimental period, with emphasis on potential respiratory disorders (coughing, sneezing and thumping) as well as systemic signs (depression, reluctance to move and fever). Specifically, rectal temperatures were taken on days $1,2,3,5$ and 7 after both viral inoculations, and also after 10 and 15 days postSwH1N1 inoculation.

Nasal swabs were taken on days 1, 2, 4, 6, 10, 21, 22, 23, 25 and $28 \mathrm{PI}$, placed in $1 \mathrm{~mL}$ of PBS and frozen at $-80{ }^{\circ} \mathrm{C}$ until further use. Complete necropsy was done on each animal, with special emphasis on the respiratory tract. Gross lung lesions were assessed for the presence or absence of pulmonary cranioventral multifocal consolidation and when present, extension was recorded. Right lung was used to perform a broncho-alveolar lavage (BAL) using $200 \mathrm{~mL}$ of PBS (animals from groups Mock/Mock and 
Table I. Primers and probes used for TaqMan one-step qRT-PCR.

\begin{tabular}{lcccc}
\hline IAV & Primer/probe & $\begin{array}{c}\text { Concentration } \\
(\mu \mathrm{M})\end{array}$ & Sequence $\left(5^{\prime}-3^{\prime}\right)$ & Reference \\
\hline $\begin{array}{l}\text { European } \\
\text { avian-like } \\
\text { swine }\end{array}$ & M+25 & 0.9 & AGA TGA GTC TTC TAA CCG AGG TCG & \\
& M+64 E-A-L-S & 1.8 & TGC AAA RAC AYC TTC CAG TCT CTG & This study \\
& 0.4 & FAM $^{\mathrm{a}}$ - TCR GGC CCC CTC AAA GCC GA- & This study \\
A(H1N1) & M+25 & 0.9 & AGA TGA GTC TTC TAA CCG AGG TCG & {$[16]$} \\
2009 & M-124 human09 & 0.9 & TGC AAA GAC ACT TTC CAG TCT CTG & This study \\
& M+64 & 0.2 & FAM- TCA GGC CCC CTC AAA GCC GA- & {$[16]$} \\
& & & TAMRA & \\
\hline
\end{tabular}

${ }^{a}$ FAM, 6-carboxylfluorescein.

b TAMRA, 6-carboxyltetramethyl rhodamine.

Mock/pH1N1) and the left one sampled for histopathological and virological studies (animals from all experimental groups). Specifically, samples from lung (apical, middle and diaphragmatic lobes), nasal turbinate, nasal septae, trachea and tonsil were collected and fixed by immersion in $10 \%$ buffered formalin. Additional lung tissues (apical and cardiac lobes) were frozen at $-80{ }^{\circ} \mathrm{C}$ until their use for viral RNA extraction.

\subsection{Pathological procedures}

Fixed tissue samples were dehydrated through graded alcohols, embedded in paraffin and stained with hematoxylin-eosin. Each tissue was microscopically studied for the presence of inflammatory lesions. In the lung, broncho-interstitial pneumonia (BIP) intensity was assessed by means a semi-quantitative scoring ( 0 to 3 , indicating lack of, mild, moderate or severe pneumonia lesions, respectively).

\subsection{Antibody detection}

Anti-influenza A virus nucleoprotein (NP) antibody levels were studied in serum using the ID Screen ${ }^{\circledR}$ Influenza A Antibody Competition ELISA (ID-Vet, Montpellier, France) following manufacturer's instructions on days 0,21 and $28 \mathrm{PI}$.

Detection of specific IgG and IgA in nasal swabs, serum and BAL was performed modifying the ID Screen ${ }^{\circledR}$ Influenza A Antibody Competition ELISA with a goat anti-porcine $\operatorname{IgG}(\mathrm{Fc}): \mathrm{HRP}$ (Serotec AAI41P) antibody at 1:100 000 dilution or a goat anti-porcine IgA:HRP (Serotec AAI40P) antibody at 1:10 000 dilution as secondary antibodies. Nasal swabs and BAL samples were used neat in the
ELISA assay whereas serum samples were used at $1 / 100$ dilution. Fifty $\mu \mathrm{L}$ of each sample or serum dilutions were used in the ELISA assay.

Hemagglutination-inhibition (HI) assay was used to measure antibody titres from serum samples at days 0,21 and 28 PI. The HI test was performed according to standard procedures [25]. The test was standardized at 4 hemmagglutinin units (HAU). To remove non-specific inhibitors of hemagglutination and natural agglutinins of sera, samples were treated with receptor-destroying enzyme (RDE) from Vibrio Cholerae (Sigma, St. Louis, MO, USA) overnight, inactivated at $56{ }^{\circ} \mathrm{C}$ for $60 \mathrm{~min}$ and adsorbed into chicken red blood cells at $50 \%$ and $4{ }^{\circ} \mathrm{C}$. The starting dilution was 1:20.

\subsection{Hydrolysis probe and primer sets}

The two influenza viruses used in this study and all the available European avian-like swine and the new human influenza virus $\mathrm{A}(\mathrm{H} 1 \mathrm{~N} 1) 2009 M$ gene sequences were downloaded, aligned from the Influenza Viruses Resource ${ }^{2}$ and then compared to the $M$ gene primer and probe sequences previously reported [22]. Three mismatches were identified for the European avian-like SIV sequences and four in the new human influenza virus $\mathrm{A}(\mathrm{H} 1 \mathrm{~N} 1) 2009$ sequences in the target sequence of the primer M-124. Also, one mismatch was detected in the target sequence of the $\mathrm{M}+64$ probe affecting the European avian-like SIV amplification. Taking all this information into account, primers and probes were modified to improve the European avian-like SIV detection

\footnotetext{
2 http://www.ncbi.nlm.nih.gov/genomes/FLU/FLU. html.
} 
(Tab. I). Primers used for the new human influenza virus $\mathrm{A}(\mathrm{H} 1 \mathrm{~N} 1) 2009$ detection in the present work were different from those of recent reports [28], although they were also based on previously reported $M$ gene primer and probe sequences [22]. Primers and probes used in this study (Tab. I) were synthesized by Tib Molbiol (Berlin, Germany).

\subsection{Quantitative RT-PCR (RT-qPCR)}

SwH1N1 and pH1N1 viral loads in nasal swabs, lung tissue and BAL were assessed following a TaqMan one-step RT-qPCR in Fast7500 equipment (Applied Biosystems, Foster City, CA, USA). Viral RNA was extracted with QIAamp viral mini kit (Qiagen, Valencia, CA, USA) obtaining $60 \mu \mathrm{L}$ of eluted viral RNA. The $M$ fragment amplification was carried out using the primers and probe concentrations indicated in Table I and One-Step RT-PCR Master Mix Reagents (Applied Biosystems) following the manufacturer's instructions using $5 \mu \mathrm{L}$ of eluted RNA in a total volume of $25 \mu \mathrm{L}$. The amplification conditions were as follows: reverse transcription at $48{ }^{\circ} \mathrm{C}$ for $30 \mathrm{~min}$; initial denaturation reaction at $95{ }^{\circ} \mathrm{C}$ for $15 \mathrm{~min}$ and 40 PCR-cycles of $95{ }^{\circ} \mathrm{C}$ $15 \mathrm{~s}$ and $60^{\circ} \mathrm{C} 1 \mathrm{~min}$.

Standard curves and quantification were achieved by prior amplification of a $99 \mathrm{bp}$ fragment of the $M$ gene using both aforementioned strains as templates, primers described in Table I and the One-Step RTPCR reagents (Qiagen) following the manufacturer's instructions, using $5 \mu \mathrm{L}$ of eluted RNA in a total volume of $25 \mu \mathrm{L}$. The amplification conditions were the following: reverse transcription at $50{ }^{\circ} \mathrm{C}$ for $30 \mathrm{~min}$; initial denaturation reaction at $95{ }^{\circ} \mathrm{C}$ for $15 \mathrm{~min}$ and 40 PCR-cycles of $94{ }^{\circ} \mathrm{C} 30 \mathrm{~s}, 55^{\circ} \mathrm{C} 1 \mathrm{~min}$ and $72{ }^{\circ} \mathrm{C} 1 \mathrm{~min}$. The obtained $M$ gene fragment amplicon was cloned into pGEMT vector (Promega Madison, WI, USA) and transformed by heat shock in Escherichia coli competent cells (Invitrogen, Paisley, UK). The recombinant plasmid was purified using the QIA prep Spin kit (Qiagen) and spectrophotometrically quantified (Qubit, Invitrogen). The copy number of recombinant plasmids was calculated as previously described [7] following the formula: $\mathrm{N}$ (molecules per $\mu \mathrm{L})=(\mathrm{C}$ (DNA) $\mu \mathrm{g} / \mu \mathrm{L} / \mathrm{K}$ (fragment size in bp)) $\times 182.5 \times 10^{23}$ (factor derived from the molecular mass per the Avogadro constant). Serial 10 -fold dilutions of both plasmids of known concentration were made and the standard curves were generated using $1.83 \times 10^{2}$ to $1.83 \times 10^{6}$ copies of recombinant plasmid with the $M$ gene fragment from the $\mathrm{pH} 1 \mathrm{~N} 1$ and $2.26 \times 10^{2}$ to $2.26 \times 10^{6}$ of recombinant plasmid with the $M$ gene fragment from
SwH1N1. The limit of detection (LoD) for pH1N1 was 11.65 plasmid copies per reaction, which corresponded to $0.05 \mathrm{TCID}_{50}$ per reaction. In the case of the SwH1N1 the LoD was 13.71 copies per reaction, which corresponded to $0.5 \mathrm{TCID}_{50}$ per reaction. The genome equivalent copies (GEC) of plasmid from the collected samples were determined based on these standard curves and taking into account their volumes. Thus, the LoD for pH1N1 was $2.82 \log _{10}$ GEC and for SwH1N1 was $2.86 \log _{10}$ GEC per nasal swab; for pH1N1 the LoD was $4.12 \log _{10}$ GEC per gram of apical and middle lobes of lung and $2.82 \log _{10}$ GEC per $\mathrm{mL}$ of BAL. Since the efficiency of retrotranscription was not directly determined, GEC numbers did not reflect exactly the number of viral RNA molecules. The RTqPCR for SwH1N1 virus was used for samples taken between day 0 and day $21 \mathrm{PI}$ in the experimental procedure, whereas RT-qPCR for $\mathrm{pH} 1 \mathrm{~N} 1$ virus was used for samples taken between days 21 to 28 PI.

\subsection{Sequencing influenza virus}

The complete amplification of the PB1, PB2, PA, HA, NP, MP and NS segments of pH1N1 of the inoculum (GenBank accession numbers GQ464405GQ464411) before and after infection, were obtained using the genome primer set and protocol recommended by the World Health Organization (WHO) ${ }^{3}$. For SwH1N1, the complete NP segment and the $5^{\prime}$ end of the hemagglutinin (HA) segment were also sequenced before and after infection using the same set of primers. The amplified products were analyzed by agarose gel electrophoresis, stained with SYBR Gold ${ }^{\circledR}$ (Molecular Probes, Eugene, USA) and purified using the NucleoFast 96 PCR kit (MACHEREY-NAGEL GmbH \& Co KG, Düren, Germany). Sequencing of both strands was performed using the BigDye technology (Applied Biosystems), with the primers M13F (5'-TGTAAAACGACGGC CAGT- $\left.{ }^{\prime}\right)$ and M13R (5'CAGGAAACAGCTATGA CC- ${ }^{\prime}$ ). Sequence assembly was accomplished using the programs Phred [3, 4], Phrap and Consed [9], and Bioedit [10]. Homology and identity searches with respect to influenza genome sequences available at the GenBank were performed by using the BLAST utilities ${ }^{4}$.

\subsection{Statistical analysis}

The Mann-Whitney test was used to compare ELISA titres in the different samples tested, and viral

\footnotetext{
3 http://www.who.int/csr/resources/publications/ swineflu/GenomePrimers_20090512.pdf.

${ }^{4} \mathrm{http} / / /$ www.ncbi.nlm.nih.gov/BLAST.
} 
loads in BAL, nasal swabs and lungs between experimental groups. All analyses were carried out with NCSS 2004 and PASS 2005 softwares (Kavysville, Utah, USA). The significance level was set at 0.05 with statistical tendencies reported when $p<0.10$.

\section{RESULTS}

\subsection{Clinical outcome}

No relevant respiratory or systemic clinical signs were observed in any pig in any of the experimental groups. Moreover, no individual pig showed abnormal rectal temperatures $\left(\geq 40{ }^{\circ} \mathrm{C}\right.$ ) throughout the duration of the study.

\subsection{Pathological studies}

Significant gross lesions compatible with BIP were observed in 6 pigs, all of them corresponding to the Mock/pH1N1 group. Remarkably, 1 out of 2 pigs sacrificed at both 23 and 25 days PI (days 2 and 4 post-pH1N1-inoculation, respectively) had BIP-like lesions. All remaining Mock/pH1N1 pigs $(n=4)$ studied on day 28 PI also had BIP-compatible lesions (Fig. 1A). No gross lesions compatible with BIP were observed in any pig of the rest of the groups studied. Besides this, one control pig (Mock/Mock) euthanized on day $23 \mathrm{PI}$ had serous arthritis of the left coxo-femoral joint, one double inoculated animal (SwH1N1/ pH1N1) had fibrous/fibrinous polyserositis and another SwH1N1/pH1N1 pig had fibrous pleuritis and mild pulmonary cranio-ventral scars.

Microscopic pulmonary lesions were observed in all but the animals from Mock/ Mock group (Figs. 1B and 1C). These lesions consisted of BIP, characterized by attenuation and loss of the bronchiolar epithelium, with mononuclear (lymphocyte and plasma cells) infiltration in the lamina propria of bronchi and bronchioli, as well as the presence of macrophages and lymphocytes within alveoli surrounding affected bronchi. Sometimes such inflammation also affected the lung interstitium. Bronchus-associated lymphoid tissue was occasionally hyperplastic. Such BIP lesions were present in all Mock/pH1N1 pigs, with mild $(n=1)$, moderate $(n=4)$ and severe $(n=3)$ intensity. Two pigs from each of the SwH1N1/Mock and SwH1N1/pH1N1 groups also showed BIP, although intensity was lower; one pig with mild and another with moderate lesions in group SwH1N1/Mock, and one animal with mild and another with mild-to-moderate lesions in group SwH1N1/pH1N1. Average histopathological BIP score is displayed in Figure 1D. No histological relevant findings were observed in the rest of studied tissues (nasal turbinates and septae, trachea and tonsil).

\subsection{Antibody response against influenza viruses}

Total anti-NP antibody levels, independent of their isotype, were measured with the competition ELISA kit in serum from all animals at the beginning of the assay (day 0 ), just before inoculation with pH1N1 (day 21 PI) and seven days after inoculation with pH1N1 (day $28 \mathrm{PI}$ ). Results represented in Figure 2A show that all animals were seronegative at the beginning of the experiment. At day $21 \mathrm{PI}$, only the animals in the two groups infected with SwH1N1 SIV (SwH1N1/Mock and SwH1N1/pH1N1) had seroconverted and had significantly higher antibody levels in serum $(p=0.0001)$ as compared with the four $\mathrm{pH} 1 \mathrm{~N} 1$ infected pigs in the Mock/ pH1N1 group, which had total antibody levels equal or below $60 \%$ of inhibition, indicating that a primary immune response against pH1N1 was taking place.

No significant differences were observed when antibody levels from the four animals in group SwH1N1/pH1N1 were compared with those from group SwH1N1/Mock, although there was a clear statistical trend $(p=0.11)$ to suspect that $\mathrm{pH} 1 \mathrm{~N} 1$ induced a secondary response. The effect of pH1N1 infection was further evaluated comparing IgA delta values between day 21 and 28 between SwH1N1/ Mock and SwH1N1/pH1N1 groups. The $p$-value was 0.06 , indicating a strong tendency close to significance. Moreover, this tendency was also observed when serum samples were analyzed for specific IgA (Fig. 3A) and IgG (Fig. 3B) anti-NP antibodies in all the animals although to a lesser extent. 

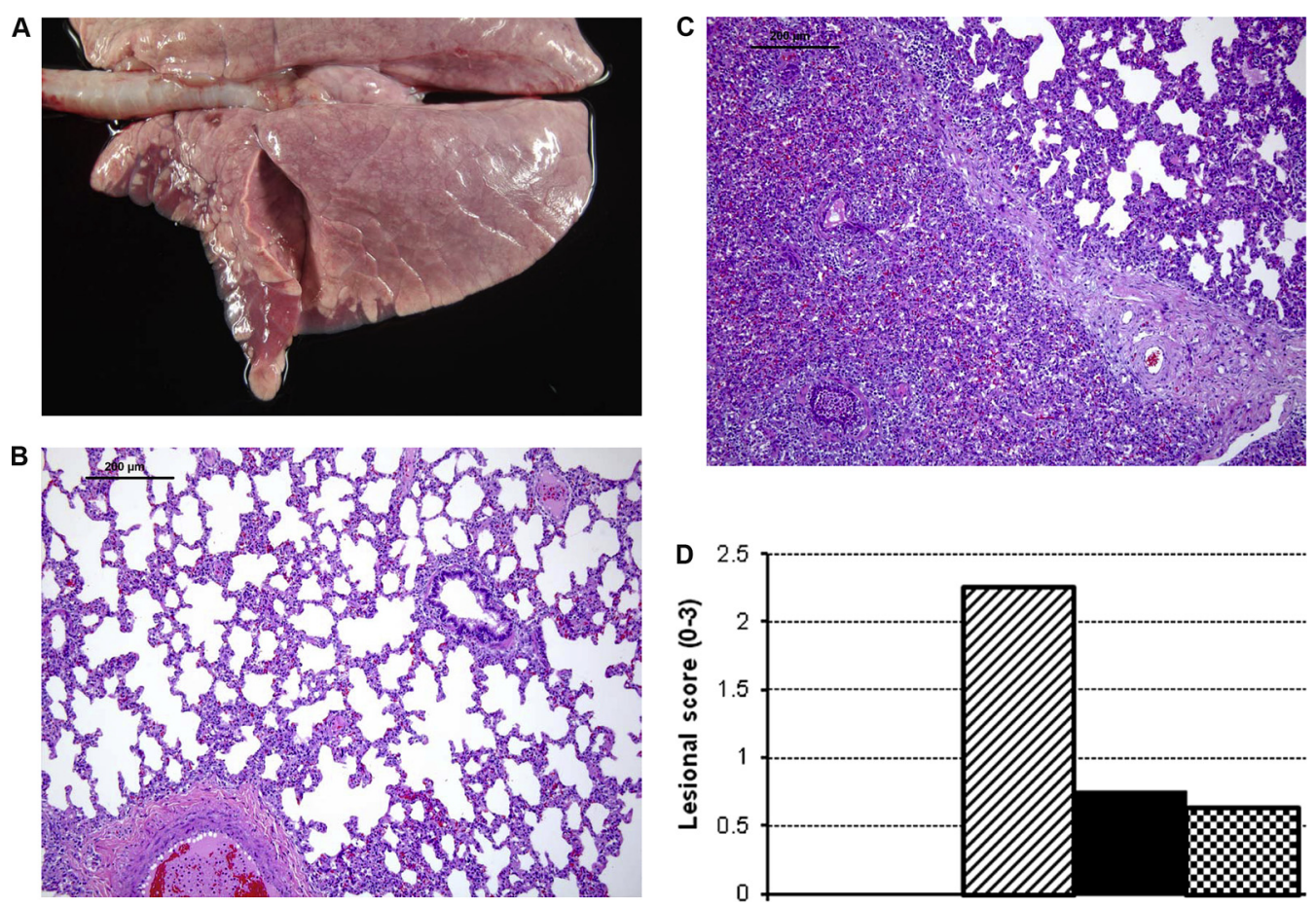

Figure 1. Pathological analysis at day 28 PI. (A) A representative picture of lung from group Mock/ pH1N1. The lungs show moderate broncho-interstitial pneumonia (BIP)-compatible lesions. (B) Lung from a Mock/Mock control pig. Normal pulmonary parenchyma. Note the minimal thickness of interalveolar walls, characterised by pneumocytes and blood capillaries. Peribronchial lymphoid tissue is minimally developed. Haematoxylin and eosin stain. Bar $=200 \mu \mathrm{m}$. (C) Lung from a pH1N1 infected pig (day 7 postpH1N1 inoculation). Marked presence of mononuclear inflammatory cells within alveoli, almost collapsing alveolar lumen. Lymphocyte perivascular cuffing together with moderate hyperplasia of the lymphoid tissue surrounding a bronchioli. Haematoxylin and eosin stain. Bar $=200 \mu \mathrm{m}$. (D) Pathological score for all the animals in the assay ( $\square$ Mock/Mock, $\mathbb{Z}$ Mock/pH1N1, SwH1N1/Mock, $\square$ SwH1N1/pH1N1). (A color version of this figure is available at www.vetres.org.)

Humoral protection against influenza virus is predominantly mediated by antibodies against HA. Thus, serum samples were examined by $\mathrm{HI}$ assays against SwH1N1 and pH1N1 (Tab. II) at day 0, 21 and 28 PI. All animals in the group infected by SwH1N1 exhibited HI antibody titres at day $21 \mathrm{PI}$, which remained constant until day 28 PI. In the case of 3 out of 4 animals in group Mock/pH1N1, some low level of HI antibodies were present at day 28 PI (7 days after pH1N1 infection). However, with one exception, HI titres elicited by SwH1N1/pH1N1 animals exhibited an increment between day 21 and
28 PI when tested against SwH1N1. Also, HI antibody titres in the SwH1N1/pH1N1 group were higher than those observed in the animals from SwH1N1/Mock group (Tab. II). All sera collected at day 21 PI remained negative for $\mathrm{HI}$ when tested against $\mathrm{pH} 1 \mathrm{~N} 1$, with the exception of animal 152. However, at day $28 \mathrm{PI}, \mathrm{HI}$ titres against $\mathrm{pH} 1 \mathrm{~N} 1$ from animals of the SwH1N1/pH1N1 group were higher than for the Mock/pH1N1 group. Surprisingly, one animal (178) gave low serum responses in ELISA and $\mathrm{HI}$ tests, but exhibited high antibody OD ratios against the NP at the nasal cavity. 

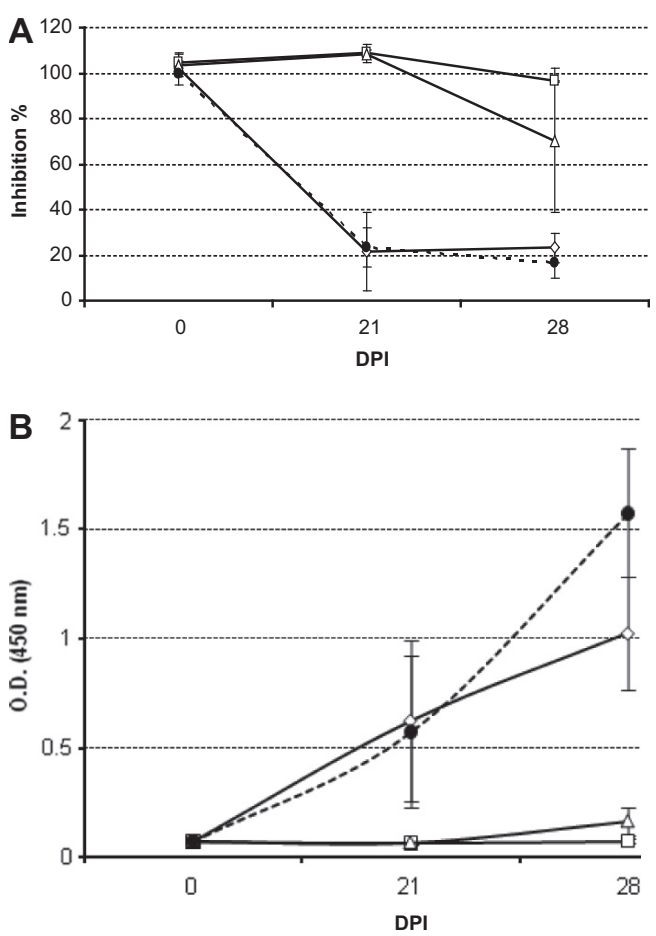

Figure 2. Antibodies in serum and nasal swabs. (A) Influenza total anti-NP antibodies in serum were analyzed by ELISA competition assay. (B) Influenza $\operatorname{IgA}$ anti-NP antibodies in nasal swabs were analyzed by ELISA at day 0,21 and 28 PI. White squares, white rhomboids, white triangles and black circles represent average values from animals belonging to Mock/Mock ( $\square)$, SwH1N1/Mock $(\diamond)$, Mock/pH1N1 $(\Delta)$ and SwH1N1/pH1N1 (O) group, respectively. Error bars represent one SD above and below the mean.

Specific $\operatorname{IgA}$ and $\operatorname{IgG}$ anti-NP antibodies were also detected in BAL of Mock/pH1N1 animals at tested times $(23,25$ and 28 days PI, which were day 2, 4 and 7 PI for $\mathrm{pH} 1 \mathrm{~N} 1$ infection) (Fig. 4).

\subsection{Viral RNA quantification}

Viral RNA levels were investigated in respiratory tissues. SwH1N1 RNA was detected in nasal swabs of pig groups SwH1N1/Mock
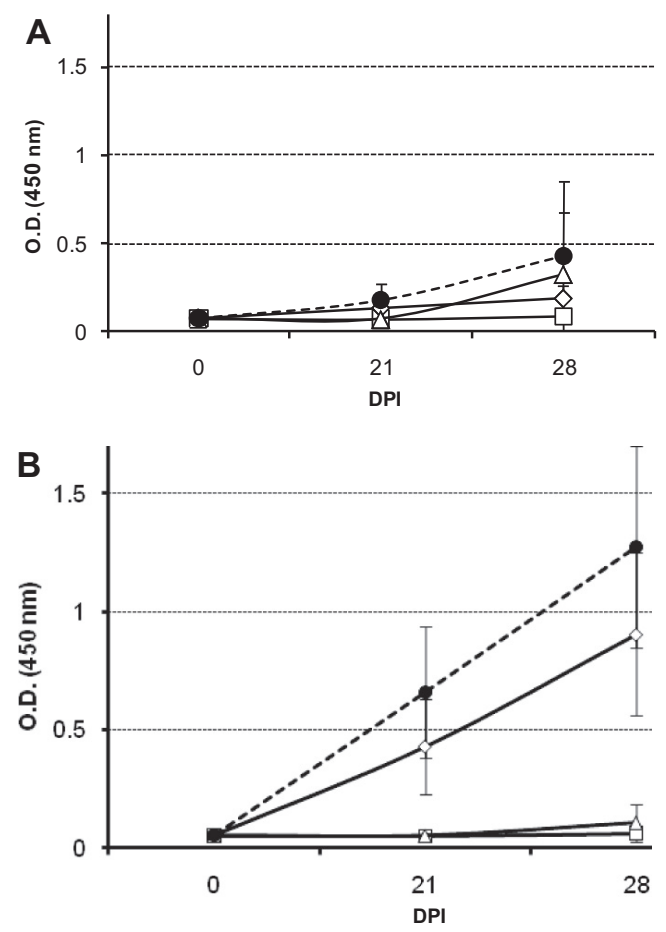

Figure 3. $\operatorname{IgA}$ and $\operatorname{IgG}$ antibodies in serum. (A) Influenza IgA and (B) IgG anti-NP antibodies in serum were analyzed by ELISA at day 0,21 and 28 PI. White squares, white rhomboids, white triangles and black circles represent mean values from animals belonging to Mock/Mock ( $\square$ ), SwH1N1/ $\operatorname{Mock}(\diamond), \operatorname{Mock} / \mathrm{pH} 1 \mathrm{~N} 1(\Delta)$ and SwH1N1/pH1N1 (๑) groups, respectively. Error bars represent one $\mathrm{SD}$ above and below the mean.

and SwH1N1/pH1N1 from day 1 to $7 \mathrm{PI}$, and had the maximum viral RNA load between days 2 and 4 PI (Figs. 5A and 5C). No viral RNA was detected on days 10 and 21 PI and in any day PI in the remaining groups (Mock/ Mock and Mock/pH1N1). Similarly, pH1N1 RNA was detected between 1 and 7 days PI with this virus, with a peak load between days 2 and 4 PI (Fig. 5B). No pH1N1 viral RNA was detected in any of the samples in group SwH1N1/pH1N1 at any of the time-points analyzed after $\mathrm{pH} 1 \mathrm{~N} 1$ inoculation or in the rest of the animal groups (Mock/Mock and SwH1N1/ 
Table II. Individual hemagglutination inhibition titres from sera belonging to animals from different infection groups (Mock/Mock, SwH1N1/Mock, Mock/pH1N1 and SwH1N1/pH1N1). Samples were tested for hemagglutinin antigens from the viruses used in the experimental infection (SwH1N1 and pH1N1).

\begin{tabular}{|c|c|c|c|c|c|c|c|}
\hline & \multirow[t]{2}{*}{ Animal number } & \multicolumn{3}{|c|}{$\begin{array}{c}\text { SwH1N1 (A/Swine/Spain/ } \\
53207 / 2004)\end{array}$} & \multicolumn{3}{|c|}{$\begin{array}{c}\text { pH1N1 (A/Catalonia/63/ } \\
\text { 2009) }\end{array}$} \\
\hline & & d0 & $\mathrm{d} 21$ & $\mathrm{~d} 28$ & d0 & $\mathrm{d} 21$ & $\mathrm{~d} 28$ \\
\hline \multirow[t]{2}{*}{ Mock/Mock } & 160 & $<20$ & $<20$ & $<20$ & $<20$ & $<20$ & $<20$ \\
\hline & 186 & $<20$ & $<20$ & $<20$ & $<20$ & $<20$ & $<20$ \\
\hline \multirow[t]{4}{*}{ SwH1N1/Mock } & 87 & $<20$ & 160 & 160 & $<20$ & $<20$ & $<20$ \\
\hline & 152 & $<20$ & 160 & 160 & $<20$ & 20 & $<20$ \\
\hline & 153 & $<20$ & 160 & 160 & $<20$ & $<20$ & $<20$ \\
\hline & 159 & $<20$ & 160 & 160 & $<20$ & $<20$ & $<20$ \\
\hline \multirow[t]{4}{*}{ Mock/pH1N1 } & 82 & $<20$ & $<20$ & $<20$ & $<20$ & $<20$ & 40 \\
\hline & 180 & $<20$ & $<20$ & $<20$ & $<20$ & $<20$ & 40 \\
\hline & 188 & $<20$ & $<20$ & $<20$ & $<20$ & $<20$ & 20 \\
\hline & 200 & $<20$ & $<20$ & $<20$ & $<20$ & $<20$ & $<20$ \\
\hline \multirow[t]{4}{*}{ SwH1N1/pH1N1 } & 84 & $<20$ & 160 & 320 & $<20$ & $<20$ & 80 \\
\hline & 86 & $<20$ & 80 & 640 & $<20$ & $<20$ & 320 \\
\hline & 168 & $<20$ & 80 & 320 & $<20$ & $<20$ & 80 \\
\hline & 178 & $<20$ & 80 & 80 & $<20$ & $<20$ & $<20$ \\
\hline
\end{tabular}

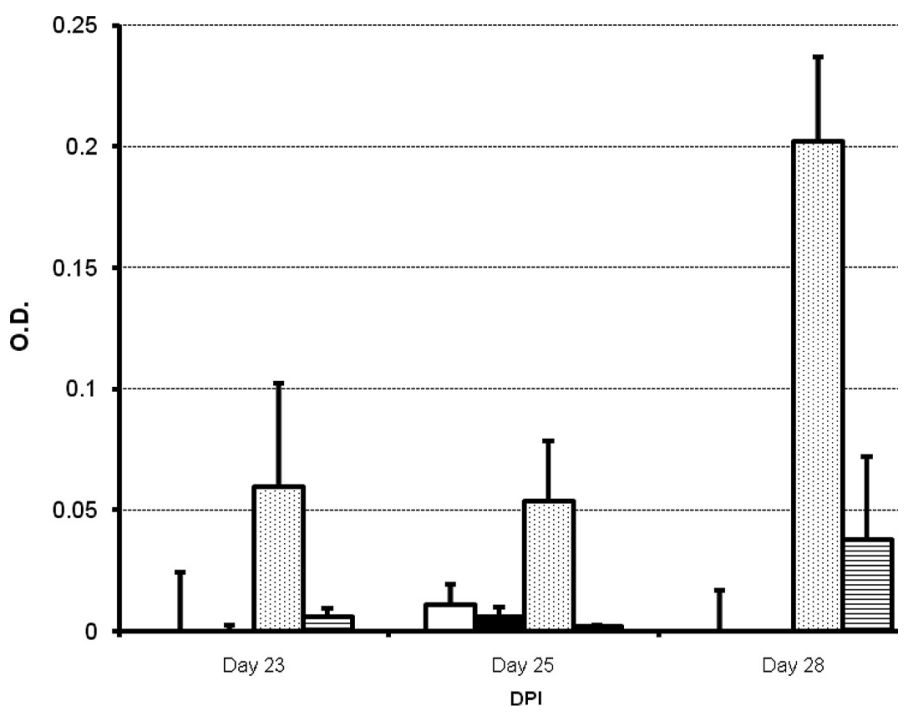

Figure 4. $\operatorname{IgA}$ and $\operatorname{IgG}$ antibodies in BAL. Influenza $\operatorname{IgA}$ and $\operatorname{IgG}$ anti-NP antibodies in BAL were analyzed by ELISA at day 23, 25 and 28 PI (corresponding to day 2, 4 and 7 after pH1N1 inoculation). White bars and dotted bars represent average IgA values of 2 and 4 animals belonging to Mock/Mock and Mock/pH1N1 groups, respectively. Black bars and lined bars represent average $\operatorname{IgG}$ values of 2 and 4 animals belonging to Mock/Mock and Mock/pH1N1 groups, respectively. Error bars represent one SD above and below the mean. 

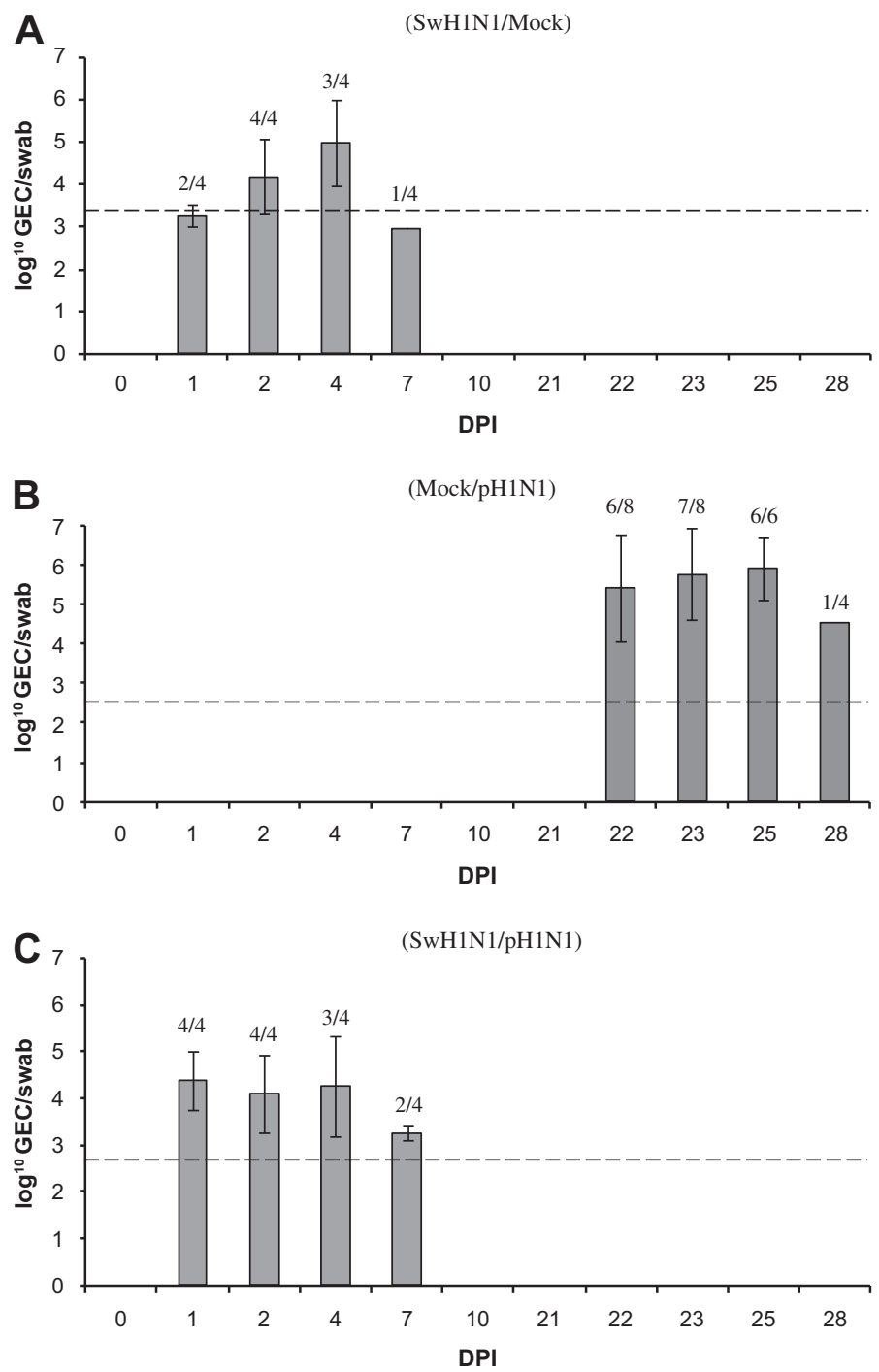

Figure 5. Influenza viral RNA load in nasal swabs. Quantification of influenza RNA was performed by RTqPCR at the indicated days PI. Bars indicate average values of positive samples in genome equivalent copies (GEC) of plasmid per swab in group SwH1N1/Mock (A), Mock/pH1N1 (B) and SwH1N1/pH1N1 (C). The number of positive samples from the total number of animals was indicated above each bar. Samples from animals in group Mock/Mock were all below the limit of detection, which is indicated by the dotted line. Error bars represent one SD above and below the mean.

Mock). Differences between GEC values in animals from group Mock/pH1N1 compared with those from SwH1N1/pH1N1 animals on days 22,23 and 25 of the experiment were statistically significant $(p=0.02 ; p=0.02$ and $p=0.004$, respectively).

Influenza RNA load was also analyzed in the lungs (apical and cardiac lobes) of animals 
A
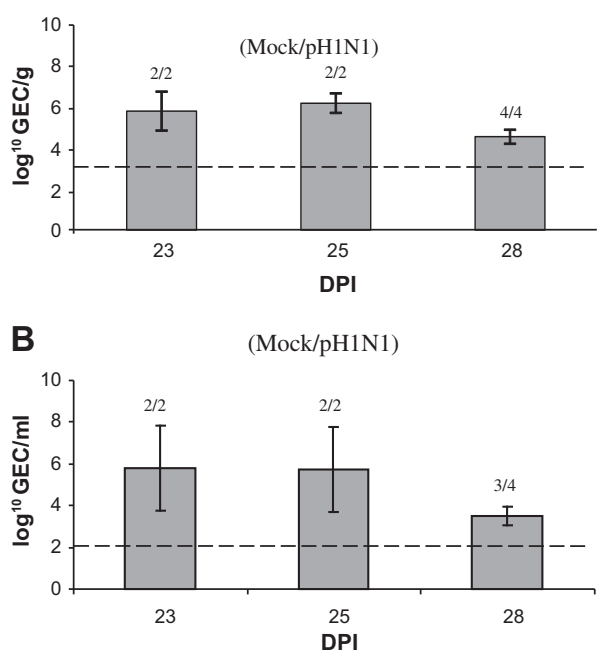

Figure 6. Influenza viral load in lung tissues and BAL. Quantitation of influenza RNA was performed by RT-qPCR at day 23, 25 and 28 PI (corresponding to day 2, 4 and 7 after $\mathrm{pH} 1 \mathrm{~N} 1$ inoculation). Bars indicate average values of positive samples in genome equivalent copies (GEC) of plasmid per swab in group Mock/pH1N1 for lung tissue (A) and BAL (B). The number of positive samples from the total number of animals was indicated above each bar. Samples from animals in groups Mock/Mock and SwH1N1/pH1N1 were all below the detection limit, indicated by the dotted line. Error bars represent one SD above and below the mean.

belonging to groups Mock/Mock, Mock/pH1N1 and SwH1N1/pH1N1 on days 23, 25 and 28 of the experiment (corresponding to day 2, 4 and 7 after pH1N1 infection) (Fig. 6A). No viral RNA was detected in the lungs of any animal belonging to the Mock/Mock group or SwH1N1/pH1N1 group in any lung tissue tested. Differences between viral RNA load in animals from group Mock/pH1N1 compared with values from lung from group $\mathrm{SwH} 1 \mathrm{~N} 1 / \mathrm{pH} 1 \mathrm{~N} 1$ on days 22,25 and 28 of the experiment were significantly different $(p=0.03)$. Influenza RNA was also detected in BAL from most of the animals in Mock/pH1N1 group (Fig. 6B) whereas no viral RNA was found in BAL from animals in Mock/Mock group.

\subsection{Sequencing of SwH1N1 and pH1N1}

The complete NP segment and the 5' end of HA sequences of SwH1N1 were analyzed and showed $100 \%$ identity to those reported previously (CY010583 and CY010580). Secondly, sequences of the complete PB2, PB1, PA, $\mathrm{HA}, \mathrm{NP}, \mathrm{MP}$ and NS genes of pH1N1 used for infection showed no variation when compared to other pandemic viruses. Furthermore, the sequences of $\mathrm{pH} 1 \mathrm{~N} 1$ isolated after pig infection were identical to those of the $\mathrm{pH} 1 \mathrm{~N} 1$ inoculum. Comparison of amino acid sequences from SwH1N1 with $\mathrm{pH} 1 \mathrm{~N} 1$ sequences resulted in an NP homology of $92 \%$ whereas in the case of HA this value was $78 \%$ and for NA it was $96 \%$.

\section{DISCUSSION}

In the present study, the possible protective effect of an infection with a circulating European avian-like swine A/Swine/Spain/53207/ 2004 (H1N1) influenza virus (referred to as SwH1N1) in colostrum-deprived pigs was evaluated against a subsequent infection with the new human A/Catalonia/63/2009(H1N1) influenza virus (referred to as $\mathrm{pH} 1 \mathrm{~N} 1$ ). A prior exposure to the SwH1N1 strain induced immunity able to substantially reduce if not inhibit pH1N1 shedding and viral RNA load in respiratory tissues after a subsequent $\mathrm{pH} 1 \mathrm{~N} 1$ infection even in the absence of detectable cross-HI antibodies. It also protected pigs from lesion development. Additionally, previous results from studies of pigs infected with the new human A(H1N1) 2009 influenza virus were confirmed in the present work. The results presented here show that intranasal infection of pigs with $10^{6.15} \mathrm{TCID}_{50}$ of $\mathrm{pH} 1 \mathrm{~N} 1$ virus exhibited mild to moderate lung lesions, resulting in virus shedding and activation of a specific humoral immune response, as it has been previously shown [16].

The typical influenza-like symptoms were not observed in either group Mock/pH1N1 or SwH1N1 infected animals used in the present experiment. Such a situation should not be surprising, since clinical signs including fever 
have only been reported when intratracheal inoculation was performed with a high virus dose $\left(\geq 7.5 \log _{10} \mathrm{TCID}_{50}\right)[23,24]$. Therefore, the experimental conditions used here induced a subclinical infection, a situation very often encountered on farms as most of SIV infections in field conditions cause subclinical infections [14].

After infection, virus RNA load in nasal swabs was detected very rapidly (at day $1 \mathrm{PI}$ ) not only for SwH1N1 but also for pH1N1. These results are in line with previous data showing that pigs are susceptible to the novel influenza virus A(H1N1) 2009 and they are able to infect contact pigs as soon as 3 days PI [16]. They also support data obtained from a naturally infected swine herd [28]. Thus, it is conceivable that this virus would probably spread quickly and efficiently if introduced into SIV H1N1 free farms. Reports from 19 countries world wide ${ }^{1}$ about putative humanto-pig transmissions and also experimental studies of sequential passages of the virus in pigs [1] support this observation. However, the data presented in this work indicated that a previous infection with at least one circulating European avian-like H1N1 SIV strain would decrease the risk of a further infection with $\mathrm{A}(\mathrm{H} 1 \mathrm{N1})$ 2009, and consequently, the potential of further reassorting.

Antiviral adaptive immune mechanisms against influenza virus involve neutralizing antibodies, including secretory IgA at mucosal surfaces, and CTL. Interestingly, primary influenza virus infection in pigs induced local antigenspecific lymphoproliferative responses and a long-lived increase of lung $\mathrm{CD} 8^{+} \mathrm{T}$ cells which could play a role in the broad-spectrum immune protection to heterotypic virus strains [11]. However, humoral protection against influenza viruses is mainly mediated by antibody responses to HA [17]. In a previous study, pigs immunized as a result of intranasal inoculation with either $\mathrm{H} 1 \mathrm{~N} 1$ or $\mathrm{H} 3 \mathrm{~N} 2$ showed partial clinical protection against $\mathrm{H} 1 \mathrm{~N} 2$ challenge, and nasal and virus shedding was two days shorter than in naïve pigs [20]. Later, Heinen et al. [11] reported some heterotypic immunity in animals infected by aerosol firstly with H1N1 and later with H3N2. However, no cross-reactive HI antibodies from SIV H1N1-infected pigs were observed with H3N2 SIV or vice versa after primary infection. A recent report on serologic cross-reactivity with A (H1N1) 2009 in pigs serum showed that consecutive infection with two European SIV subtypes induced HI crossreacting antibodies to $\mathrm{A}(\mathrm{H} 1 \mathrm{~N} 1)$ influenza virus, even though European viruses do not contain a classical swine H1 HA. These authors also described that $\mathrm{HI}$ antibodies induced by a single infection with European subtype H1N1 or H1N2 SIV did not cross-react with the pandemic (H1N1) 2009 virus [15]. The results presented in this work are in full agreement with all these previous data as no cross-reactive antibodies were detected between SwH1N1 and pH1N1 before $\mathrm{pH} 1 \mathrm{~N} 1$ infection. These results suggest that sequence divergence of around $20-30 \%$ in amino acids in proteins such as HA or NA do not interfere with the generation of memory cross-reactive B cells.

Furthermore, the sequences of $\mathrm{pH} 1 \mathrm{~N} 1$ inoculated and $\mathrm{pH} 1 \mathrm{~N} 1$ isolated from infected pigs were identical, suggesting that the virus did not evolve within the study period. This is in agreement with data obtained so far in humans where genetic variability of the virus is very limited. However, recent information from a naturally infected swine herd with $\mathrm{A}(\mathrm{H} 1 \mathrm{~N} 1)$ 2009 suggested that either a higher than normal mutation rate, strong positive selective pressure, or a combination of both might apply for this infection [28]. Further work will elucidate the genetic evolution of this new virus.

Davenport et al. [2] first described in 1953 the phenomenon of original antigenic sin. This observation was later expanded showing that the phenomenon of original antigenic sin responses to influenza viruses existed not just in humans but in other species as well $[5,6$, 26]. In the context of sequential infection with two influenza viruses, the primary exposure induces proliferation of $\mathrm{B}$ cells that are either specific for the first virus only or cross-reactive with both viruses. Upon exposure to the second virus, memory $\mathrm{B}$ cells cross-reactive to both viruses outcompete naïve B cell clones just specific for novel epitopes from the second virus. Indeed, this theoretical explanation would fit with the results obtained in the present study, showing that original antigenic sin also takes 
place for SIV infection in pigs. Cross-reactive memory B cells for SwH1N1 and pH1N1 might be generated upon infection with SwH1N1 by a single SwH1N1 exposure. The fact that cross-reacting antibodies were undetected before $\mathrm{pH} 1 \mathrm{~N} 1$ infection indicated that this cross-reactive memory $\mathrm{B}$ cell population was a minor population. These memory cells would be later activated by differentiating into plasma B cells when $\mathrm{pH} 1 \mathrm{~N} 1$ infection took place, explaining the rapid increase in $\mathrm{pH} 1 \mathrm{~N} 1$ specific $\mathrm{HI}$ antibodies 7 day after $\mathrm{pH} 1 \mathrm{~N} 1$ infection in animals from group SwH1N1/pH1N1 compared with $\mathrm{HI}$ antibodies titres in animals only infected with $\mathrm{pH} 1 \mathrm{~N} 1$ (primary infection) and the higher values of $\mathrm{HI}$ antibodies against SwH1N1 at day 28 (Tab. II).

In the present experiment, the boosting effect of pH1N1 infection in group SwH1N1/ $\mathrm{pH} 1 \mathrm{~N} 1$ for IgA levels at mucosal sites and the HI cross-reactive antibodies generated after challenge would suggest that humoral responses with cross-neutralizing activity in respiratory tissues and sera played a major role in conferring protective immunity. Therefore, it seems that specific humoral immunity, particularly the one induced at the respiratory mucosa, correlated with protection in animals challenged with a subsequent infection with $\mathrm{pH} 1 \mathrm{~N} 1$.

To the authors' knowledge, this is the first study in which immunity generated against a circulating European avian-like swine (H1N1) influenza virus in pigs has been evaluated against a subsequent infection with a human (H1N1) influenza virus in pigs. In summary, a previous infection with one of the European circulating H1N1 SIV in the field was able to confer protective immunity to pigs against a challenge with the new variant $\mathrm{A}(\mathrm{H} 1 \mathrm{~N} 1)$ 2009. These data pave the way for understanding cross-protective immune responses generated between different influenza viral infections, within the same subtype or different subtypes. Further characterizations are required to understand the whole picture and all the fine mechanisms involved in cross-protection.

Acknowledgements. This work was partly funded by projects no. CSD 2006-00007 (PORCIVIR, program CONSOLIDER-INGENIO 2010) and AGL2006-
13809-C03-01 and ALG2009-12945-C02-01 by the Spanish Government and by FP-7-2008-1, 228394, NADIR project funded by the EU. Authors are very grateful to HIPRA Laboratories S.A. for generously donating the H1N1 European avian-like swine A/ Swine/Spain/53207/2004 (H1N1) influenza virus. They are also very grateful to Dr Tomás Pumarola and Dr Andrés Antón from Hospital Clinic (Barcelona) for allowing $u$ s to isolate the $\mathrm{A} /$ Catalonia/63/2009(H1N1) influenza virus from a patient sample. Authors also thank all the personnel at the biosafety level 3 facilities at CReSA and Dr Kevin Dalton for reviewing the manuscript. PhD studies of E. Crisci and G.E. Martin-Valls are founded by a doctoral FPI grant from the Spanish Ministry of Science and Innovation. PhD studies of M. Simon-Grifé are founded by a pre-doctoral FI grant of the Government of Catalunya (Spain). PhD studies of $\mathrm{T}$. Mussà are founded by a doctoral grant from the AECID (Agencia Española de Cooperación Internacional y Desarrollo).

\section{REFERENCES}

[1] Brookes S.M., Irvine R.M., Nunez A., Clifford D., Essen S., Brown I.H., et al., Influenza A (H1N1) infection in pigs, Vet. Rec. (2009) 164:760-761.

[2] Davenport F.M., Hennessy A.V., Francis T. Jr, Epidemiologic and immunologic significance of age distribution of antibody to antigenic variants of influenza virus, J. Exp. Med. (1953) 98:641-656.

[3] Ewing B., Green P., Base-calling of automated sequencer traces using phred. II. Error probabilities, Genome Res. (1998) 8:186-194.

[4] Ewing B., Hillier L., Wendl M.C., Green P., Basecalling of automated sequencer traces using phred. I. Accuracy assessment, Genome Res. (1998) 8:175185.

[5] Fazekas de St Groth S., Webster R.G., Disquisitions on original antigenic sin. II. Proof in lower creatures, J. Exp. Med. (1966) 124:347-361.

[6] Fazekas de St Groth S., Webster R.G., Disquisitions of original antigenic sin. I. Evidence in man, J. Exp. Med. (1966) 124:331-345.

[7] Fronhoffs S., Totzke G., Stier S., Wernert N., Rothe M., Bruning T., et al., A method for the rapid construction of cRNA standard curves in quantitative real-time reverse transcription polymerase chain reaction, Mol. Cell. Probes (2002) 16:99-110.

[8] Garten R.J., Davis C.T., Russell C.A., Shu B., Lindstrom S., Balish A., et al., Antigenic and genetic characteristics of swine-origin 2009 A(H1N1) influenza viruses circulating in humans, Science (2009) 325:197-201. 
[9] Gordon D., Abajian C., Green P., Consed: a graphical tool for sequence finishing, Genome Res. (1998) 8:195-202.

[10] Hall T.A., BioEdit: a user-friendly biological sequence alignment editor and analysis program for Windows 95/98/NT, Nucl. Acids Symp. (1999) 41:9598.

[11] Heinen P.P., de Boer-Luijtze E.A., Bianchi A.T., Respiratory and systemic humoral and cellular immune responses of pigs to a heterosubtypic influenza A virus infection, J. Gen. Virol. (2001) 82:26972707.

[12] Ito T., Couceiro J.N., Kelm S., Baum L.G., Krauss S., Castrucci M.R., et al., Molecular basis for the generation in pigs of influenza A viruses with pandemic potential, J. Virol. (1998) 72:7367-7373.

[13] Itoh Y., Shinya K., Kiso M., Watanabe T., Sakoda Y., Hatta M., et al., In vitro and in vivo characterization of new swine-origin H1N1 influenza viruses, Nature (2009) 460:1021-1025.

[14] Kothalawala H., Toussaint M.J., Gruys E., An overview of swine influenza, Vet. Q. (2006) 28:46-53.

[15] Kyriakis C.S., Olsen C.W., Carman S., Brown I.H., Brookes S.M., Doorsselaere J.V., Reeth K.V., Serologic cross-reactivity with pandemic (H1N1) 2009 virus in pigs, Europe, Emerg. Infect. Dis. (2010) 16:96-99.

[16] Lange E., Kalthoff D., Blohm U., Teifke J.P., Breithaupt A., Maresch C., et al., Pathogenesis and transmission of the novel swine origin influenza virus A/H1N1 after experimental infection of pigs, J. Gen. Virol. (2009) 90:2119-2123.

[17] Murphy B.R., Clements M.L., The systemic and mucosal immune response of humans to influenza A virus, Curr. Top. Microbiol. Immunol. (1989) 146:107-116.

[18] Myers K.P., Olsen C.W., Gray G.C., Cases of swine influenza in humans: a review of the literature, Clin. Infect. Dis. (2007) 44:1084-1088.
[19] Reed L.J., Muench H., A simple method of estimating fifty per cent endpoint, Am. J. Hyg. (1938) 27:493-497.

[20] Reeth K.V., Brown I., Essen S., Pensaert M., Genetic relationships, serological cross-reaction and cross-protection between $\mathrm{H} 1 \mathrm{~N} 2$ and other influenza A virus subtypes endemic in European pigs, Virus Res. (2004) 103:115-124.

[21] Smith G.J., Vijaykrishna D., Bahl J., Lycett S.J., Worobey M., Pybus O.G., et al., Origins and evolutionary genomics of the 2009 swine-origin H1N1 influenza A epidemic, Nature (2009) 459:1122-1125.

[22] Spackman E., Senne D.A., Myers T.J., Bulaga L.L., Garber L.P., Perdue M.L., et al., Development of a real-time reverse transcriptase PCR assay for type A influenza virus and the avian $\mathrm{H} 5$ and $\mathrm{H} 7$ hemagglutinin subtypes, J. Clin. Microbiol. (2002) 40:3256-3260.

[23] Sreta D., Kedkovid R., Tuamsang S., Kitikoon P., Thanawongnuwech R., Pathogenesis of swine influenza virus (Thai isolates) in weanling pigs: an experimental trial, Virol. J. (2009) 6:34.

[24] Van Reeth K., Labarque G., De Clercq S., Pensaert M., Efficacy of vaccination of pigs with different H1N1 swine influenza viruses using a recent challenge strain and different parameters of protection, Vaccine (2001) 19:4479-4486.

[25] Webster R., Krauss S., World Health Organization Manual on Animal Influenza Diagnosis and Surveillance, in: W.H.O. (Ed.), Geneva, Switzerland, 2002.

[26] Webster R.G., Original antigenic sin in ferrets: the response to sequential infections with influenza viruses, J. Immunol. (1966) 97:177-183.

[27] Webster R.G., Bean W.J. Jr, Genetics of influenza virus, Annu. Rev. Genet. (1978) 12:415-431.

[28] Weingartl H.M., Berhane Y., Hisanaga T., Neufeld J., Kehler H., Emburry-Hyatt C., et al., Genetic and pathobiologic characterization of pandemic H1N1 2009 influenza viruses from a naturally infected swine herd, J. Virol. (2010) 84:2245-2256. 